\title{
BRAIN TUMOR STEM CELLS: PHENOTYPIC CHARACTERIZATION AND DIRECTED THERAPEUTIC APPROACHES
}

\section{ABSTRACT}

The review presents the current conceptions of the origin, methods of isolation and phenotypic characterization of the brain tumor stem cells. Phenotypic similarity in molecular markers between cancer and neural stem cells is shown. Therapeutic approaches of impact on the brain tumor stem cells and on the intracellular signaling pathways of cancer stem cells are described.

KEYWORDS: brain tumor stem cells, neural stem cells, malignant gliomas, CD133, nestin

Treatment of patients with oncologic diseases is now challenging The number of malignancies, including tumors of the central nervous system (CNS) increases worldwide. The CNS tumors make from $2 \%$ to $5 \%$ among human oncologic diseases, whereas glial tumors make $50 \%$ among which the most malignant forms are the $3^{\text {rd }}-4^{\text {th }}$ degree anaplasias making from 40 to $50 \%$. Average life expectancy of patients with anaplastic gliomas and glioblastomas is 14-18 months respite aggressive therapy based on surgical resection followed by irradiation and chemotherapy.

In recent time great attention of the investigators has been given to study of cells isolated from tumors and possessing stem cells properties. This gives a new insight into tumor nature and reasons of inefficiency of various cancer treatment methods as well as promotes development and exploration of more efficient ways of treatment of oncologic diseases.

The group of scientists under leadership of J. E. Dick was one of the first who in 1997 year proved the existence of cancer stem cells (CSCs). They investigated acute myeloleukosis, at which the subpopulation making $0.01-1 \%$ of the total cell population and characterized phenotypically as $\mathrm{CD} 34^{+}$, could cause leukemia during transplantation to the immune-deficient SCID and NOD-SCID mice. During transplantation of hemopoietic cells from patients with acute myeloid, acute lymphoblast or chronic myeloid leukemia such a small amount of cells appeared to be capable to initiate respective leucosis and support them in the mice-recipients [1].

The most recent data have documented the presence of cancer stem cells in the tumors of the colon, mammary gland, pancreas, liver and lung, in the mesenchymal tumors and brain tumors (gliomas, medulloblastomas, ependymomas etc.) [2-5]. Until recently it has been thought that the number of CSCs in many malignancies makes $0.01 \%$ $-1 \%$ of the overall population of cancer cells. However as more experimental investigations have been performed their data showed that the number of CSCs in different types of cancer varies from $1 \%$ to $20 \%$ and even more in some cases.

The brain tumor stem cells (BTSCs) possess an indefinite capacity for self-maintenance, tumor generation during ortotopic implantation, genetic disorders and formation of cancer cells [6]. BTSCs possess great capacity for invasion, stimulate formation of the blood vessels and initiate cell migration [2, 3]. Apart from this, they are involved in the stimulation of cancerogenesis: there appear more and more corroborations that these cells promote cancer progression [7] and metastasis [8]. This type of cells is responsible for chemo- and radio resistance, post-surgery and radiotherapy relapse.

\section{ORIGIN, ISOLATION AND PHENOTYPIC CHARACTERISTICIS \\ OF THE BRAIN TUMOR STEM CELLS}

At the present stage of investigations there remains a disputable issue concerning the origin of the BTSCs. The neural stem cells (NSCs), the neuronal progenitor cells, and the differentiated glioma cells have been considered as the sources of the BTSCs. Thus, Shiras A. et al. (2007) confirmed spontaneous transformation of CD133+ NSCs into glioma NSCs [9]. According to Masui K. et al., the glial cells-precursors give rise to the oligodenroglial tumors involving PDGFR-associated pathways [10]. Junier M. and Sharif A. (2011) demonstrated that under influence of transforming growth factor TGF-a, which is expressed at early stages of tumor progression, the astrocytes can acquire the phenotype of neuronal progenitor cells, which under irradiation impact acquire malignant properties that, in the authors opinion, can evidence for the possibility of malignant transformation into mature glial cells and formation from them of the glioma stem cells [11]. The possibility of transformation of the differentiated glioma cells into CSCs under hypoxic conditions was demonstrated by X. Yuan et al. [5].

Several methods of obtaining and identification of CSCs, including BTSCs, have been described, among them: 
- $\quad$ analysis of the expression of CD133 molecule (prominin-1);

- identification of the cells of «side population» (SP); and

- $\quad$ formation of the neurospheres in cell culture in vitro and possibility of CSCs to form tumor in the immune-deficient animals being histologically identical to primary tumor.

Prominin-1 was first detected on the hemopoietic stem cells of the stem CD34-positive cells obtained from fetal liver and later from umbilical cord blood and peripheral blood and embryonal and adult brain. The function of prominin-1 is not well known. However during differentiation of stem cells the given membrane protein is not detected. One-nucleotide deletion of prominin- 1 is responsible for the hereditary form of retina degeneration.

CD133 is the transmembranous glycoprotein with five transmembranous domains and two large N-glicolized extracellular loops, which is localized on the plasma membrane protrusions and micro-domains. Despite the unknown cellular function of prominin-1, it is the marker of many SCs that have been identified until now, including CSCs of the gliomas, lungs, liver, prostate etc. $[4,12]$. Thus, when using the flow cytometer, it is possible to isolate the BTSCs by CD133 expression.

Notably, the number of $\mathrm{CD} 133^{+}$cells in tumor tissue can be identified not only with flow cytometry but also by immunohistochemical assay. Thus using laser flow cytometry, S.K. Singh et al. demonstrated [13] that the number of $\mathrm{CD} 133^{+}$cells makes from 0.1 to $46.8 \%$ in the glioblastomas, $6.1-45.4 \%$ in the medulloblastomas, and 3.5-37.1\% in the pilocytic astrocytomas. Other authors [14] who used immunohistochemical analysis revealed the high tissue contents (over $60 \%$ ) of $\mathrm{CD}_{133^{+}}$cells among glial tumors of various degree of anaplasia: in $29.2 \%$ of the glioblastomas, in $18.2 \%$ of the anaplastic astrocytomas, in $83.3 \%$ of the malignant oligodenrogliomas, in $6.7 \%$ of the ependimomas, in $0 \%$ of the astrocytomas, in $50 \%$ of the oligodendroastrocytomas and in $9.1 \%$ of the oligodendrogliomas. The absence or insignificant (less than $4 \%$ ) number of $\mathrm{CD}_{133^{+}}$cells was revealed in $10.4 \%$ of the glioblastomas, in $18.2 \%$ of the anaplastic astrocytomas and in $0 \%$ of the malignant oligodendrogliomas. In the malignant gliomas the $\mathrm{CD}_{133^{+}}$cells are represented by subpopulation of small cells with blue cytoplasm, capable for 3D growth in the round neurospheres.

The data concerning the prognostic value and correlation between level of $\mathrm{CD}_{133^{+}}$cells and the survival term of glioma-affected patients are contradictory so far. Thus the data obtained by Pallini $R$. et al. (2011) who investigated CD133 expression in 44 glioblastomas (GBMs) and found that presence of $2 \%$ of CD133+ cells and CD133/ Ki67 co-expression were correlated with unfavorable survivorship outcomes in patients with brain tumors [15]. Zeppernick F. et al. [2008] revealed that the presence of CD133 clusters and large number of $\mathrm{CD}_{133^{+}}$cells correlated with short survival term [16]. In their study of CD133 expression using PCR, Ma Y. H. et al. (2008) showed much higher level of $\mathrm{CD}_{133^{+}}$cells in tumor tissue compared with normal brain tissue [17]. However Kim et al. (2012) did not find any differences between level of $\mathrm{CD} 133^{+}$cells and survival term of patients with multiforme glioblastoma [18].

Depending on the number of CD133+ cells, the multiforme glioblastomas display a different phenotype. Thus multiforme glioblastomas with low content of $\mathrm{CD}_{133^{+}}$cells (less than $3 \%$ ) tend to localize in the deeper hemispheral structures and show more invasive growth involving brain ventricles and progression while on chemotherapy. These glioblastomas have the profile characteristic of the mesenchymal and proliferative subtypes. At the same time the multiforme glioblastoma with high content of $\mathrm{CD}_{133^{+}}$cells have proneural phenotype.

Notably, the CSCs isolated from tumor tissue of the CNS or from human tumor cell line following their injection to immune-deficient animals cause formation of tumor histologically identical to primary tumor. It has been shown that injection of CD133+ SCs isolated from the medulloblastoma to nude mice caused formation of the medulloblastoma. Isolation of CD133 cells with glioblastoma followed by their injection to nude mice promoted tumor formation in 10 of 10 animals.
Interestingly, the formation of identical tumor following SCs injection to the immune-deficient animals depended on the number CD133+ cells. With a small (up to $1 \%$ ) initial tumor content of $\mathrm{CD} 133^{+}$cells the development of a small-size tumor was registered in about one-third of the experimental animals. Increase of tumor content of CD133+ cells to $30 \%$ promoted almost $100 \%$ tumor renewal.

Identification of CSCs by assessing «side population» is based on the passive uptake of Hoechst 33342 dye by live cells at laser excitation length $366 \mathrm{~nm}$. The protein-coding genes ABCG2, ABCB1 and $A B C C 1$ were found in the tumor cells which accomplish transport of both hydrophobic and hydrophilic compounds from cells into the medium. These proteins provide uptake of Hoechst or rhodamine and allow identify cells as a «side population». By the ability of cells to exclude Hoechst 33342 among cancer cells SP was found in 15 of 23 specimens of the neuroblastomas. SP cells were able to proliferate in vitro, divide asymmetrically and form neurospheres. When exploring glioma C6 cell line the SP cells were able to initiate tumor formation and reproduce full tumor phenotype that evidences for the presence in SP cells of the main properties of the stem cells, namely their ability to self-renewal and differentiation. Furthermore, only SP cells were able to form the neurospheres that is one of the most essential signs of the CSCs and differentiate into the neuronal lines.

The methods of BTSCs culture are similar to the NSCs culture methods. This exemplifies cultivation in serum-free medium in the presence of growth factors rhEGF, rhFGF-b or B-27 [12, 19]. When cultured under given conditions part of the CSCs of the intracerebral tumors form neurospheres. Thus during analysis of the gliomas having various degree of anaplasia the neurospheres were registered in 11.7 $\%$ of the glioblastomas, in $5.8 \%$ of the anaplastic oligodendrogliomas and in $29.4 \%$ of the anaplastic astrocytomas [20]. However, other authors detected formation of neurospheres in $100 \%$ of the cases during culturing of the glioblastomas and medulloblastomas in serum-free culture medium in the presence of growth factors. In the process of culturing of CSCs of the glial tumors the above investigators observed an altered number of $\mathrm{CD} 133^{+}$cells and their differentiation markers. Increase in the number of $\mathrm{CD}_{133^{+}}$cells from $3.65 \%$ to $14.6 \%$ and nestin-positive cells from $28.9 \%$ to $45.4 \%$ was demonstrated on 7 th to 21 st days of cultivation [20].

There are literature data describing the factors which stimulate and depress the number of $\mathrm{CD}_{133^{+}}$cells in the culture and influence on their activity. Thus epidermal growth factor (EGF) and the vascular epidermal growth factor (VEGF) stimulate the number of CD133+ cells in the culture as well as enhance expression of MMP and IMMP in particular, thus promoting quicker tumor growth upon injection of the activated cells to immune-deficient animals. On the contrary, depression of L-ICAM adhesion molecules expression, which is linked with transcriptional factors Oligo2 and Oligo21 regulating apoptosis and express on the surface of $\mathrm{CD}_{133^{+}}$cells leads to apoptosis and inhibition of tumor growth [21].

It is noteworthy that CD133 expression is characteristic of NSCs as well. Pallini R. et al. (2011) have demonstrated that among CD133+ cells in the glioblastoma 20 to $60 \%$ of them are CD133+CD45- NSCs and their increased number favorably influences on the survival of patients with recurrent multiforme glioblastoma. The NSCs migrate into tumor tissue and manifest antitumor properties at the expense of the suppressing factors, bone morphogenic proteins 7 (BMP-7) in particular, which belongs to the family of TGF- $\beta$ cytokines. Under the influence of BMP-7 the glioma stem cells carrying respective receptor on their surface, proliferate to lesser degree and are differentiated by the astrocyte or neuronal type that reduces their tumorigenic properties.

Until present the panel of specific BTSCs markers has not been elucidated. It has been shown that stem cells isolated from the gliomas express CD133, CD105, CD90, CD15, CD24, CD20, CD44, Nanog, Oct3/4, CXCR4 (CD184) and NF (neurofilament protein), GAPDH (glyceraldehyde-3-phosphate dehydrogenase). In addition, the BTSCs express 
markers which are characteristic of the NSCs, such as GFAP (glial fibrillary acid protein), nestin, Sox-2, Misashi-1 and Bmi-1 but they do not express early (Tuj1) and late (NeuN) neuronal markers and oligodendroglial marker Olig-1 [22]. During culturing the BTSCs can differentiate and acquire markers of the neuronal, astrocyte and oligodendroglial lines. Thus along with determination of CD133 molecule, detection of other molecules of differentiation are important for the SCs.

CD15 is the cluster of the differentiation which is identified in various normal tissues and in various types of cancer, including gliomas [23], and is viewed as the marker of BTSCs. Implantation of glioma CD15-positive cells into murine brain induces formation of new tumors [24].

CD44 is the glycoprotein, which is the receptor for hyaluronic acid, the main component of extracellular matrix, itself or in combination with other surface markers, is used for isolation of cells having properties of stem cells from various tumor types, including cancer of the mammalian gland, prostate, intestine, pancreas and squamous cell carcinomas of the head and neck. As a result of hyaluronan binding, CD44 activates many receptor thyrosine-kinases, including EGFR and ErbB2, in many cancer types, thus increasing proliferation and survival via activation of MAPK and PI3K/Akt pathways, respectively.

CD90 has been identified as a marker of the hematopoietic, mesenchymal and liver stem cells. High frequency of CD90+ cells is shown at malignant gliomas and their absence at benign astrocytomas and in normal tissue. $100 \%$ of $\mathrm{CD} 133^{+}$SCs express CD90 marker. The levels of CD133 and CD90 expression decrease during differentiation of BTSCs into glioma cells. The ability for neurosphere formation of $\mathrm{CD}^{+} / \mathrm{CD}_{133^{+}}$and $\mathrm{CD} 90^{+} / \mathrm{CD} 133^{-}$cells is similar and it is two times higher than in CD90-/CD133- cells [25]

A2B5 is the surface glycoside and marker of 02-A neuronal progenitor cells. Transplantation studies [26] have shown that $\mathrm{A}^{2 \mathrm{~B} 5}{ }^{+} /$ $\mathrm{CD} 133^{+} \mathrm{i} \mathrm{A} 2 \mathrm{~B} 5^{+} / \mathrm{CD} 133$ cells are able to generate tumors during transplantation in the experimental models assuming that $A B 5^{+}$cells have properties of the SCs. Studies of Piepmeier et al (1993) on the prognostic potential of $\mathrm{A}_{2} \mathrm{~B} 5^{+}$cells in gliomas have shown that $\mathrm{A} 2 \mathrm{~B} 5$ is the marker of unfavorable prognosis for glioma development [27].

The filament marker, nestin, that is expressed in the neuronal cellsprogenitors in the development and initially described as the marker of NSCs, was also identified later in the BTSCs [28]. Nestin expression in the gliomas has been associated with differentiation, improved cell migration, invasion potential and increased malignancy. The presence of nestin is identified in $46 \%$ of primary CNS tumors and its level of expression is even higher in the astrocytomas of various degree of malignancy in the comparison with normal brain tissue. There is no unanimous opinion in the literature regarding nestin expression. The majority of the investigators $[29,30]$ demonstrated an increased nestin expression under an increased malignancy degree of the astrocytes and correlation between the level of nestin expression and the survival term of the patients with gliomas. K. J. Kim et al. [31] did not established any statistically significant between the level of nestin expression, the malignancy degree of the astrocytes and survival term of the patients with brain gliomas. However, at the present stage the nestin is studied along with CD133 molecule as the marker of the BTSCs identification. Thus, at parallel immunohistochemical assay of glioma tumors the obtained results on the content of $\mathrm{CD}_{133^{+}}$and nestin-positive cells were close. The number of $\mathrm{CD}_{133^{+}}$and nestin-positive cells correlated with the survival of patients during post-operation period.

Sox2 is one of the key transcriptional factors that is responsible for maintenance of the stemness properties of cells in embryogenesis and adult organism, as well as plays role in the formation of neurons and neuroglia; and in the cancerogenesis and glioblastoma formation. Decrease of Sox2 after embryogenesis correlates with the loss of pluripotency and self-renewal whereas Sox2 suppression in the gliomas leads to the loss of tumorigenicity [32]. Amplification of Sox2 gene is observed in $14.4 \%$ of the multiforme glioblastomas and in $11.1 \%$ of the anaplastic oligodenrogliomas. A positive correlation has been established between the degree of tumor malignancy and Sox2 expression. The hypercellular and hyperproliferative zones of glioblastomas have the highest Sox2 expression. However, in other works the authors do not see any correlation between the degree of glioma malignancy and the survival term of patients and the Sox2 level [33, 34], although they demonstrated increase of Sox2 expression in the glioma tissues compared to normal brain tissue. Thus of today there is no identical vision relative prognostic value of Sox2 at the gliomas.

Podoplanin is the mucin-type of the transmembranous glycoprotein with less studied biological function. Apart from its role in the migration and invasion in the gliomas, podoplanin can play a significant role in the formation of spheroids. Increased expression of podoplanin in malignant astrocytic tumors as a novel molecular marker of malignant progression. K. Mishima et al. [35] consider that podoplanin can be the prognostic marker in the astrocytomas of high malignancy degree. They detected high expression of the podoplanin in 48 specimens of malignant astrocytomas but did not observe its expression in the astrocytomas of low degree malignancy.

The polycomb complex protein BMl- 1 is a member of the polycombgroup and a known regulator of two main pathways of tumor suppressors. The given protein suppresses INK4a gene transcription thereby promoting activation of cycline-dependent kinases Cdk2 and Cdk4 and hence proliferation. Besides, suppression of Bmi-1 in CD133+ glioma cell population reduces the number of the secondary spheres promoting thereby lowering of self-renewal [36]. Study of Bmi-1 expression in 62 oligodendroglial and 243 astrocyte tumors stage II-IV with the use of simple and multifactorial analysis has shown that high versus low level of Bmi-1 expression is poor prognosis for survival [37].

Musashi-1 belongs to the family of neural RNA-binding proteins. Hemmati H. et al. (2003) have shown that Musashi-1-positive tumor cells are capable to form neurospheres, capable for self-renewal and differentiation into various types of cells [38]. Y. H. Ma et al. [39] demonstrated increase of Musashi-1 in the glioma cells compared with normal tissue and correlation of high expression of mRNA protein with the malignancy degree. A correlation was established between Musashi-1 expression and proliferative Bmi-1 marker in malignant gliomas [40].

An increased expression of another marker of CSCs - Id1 was established in various types of cancer where it is involved in the proliferation, anaplasia, invasion, metastasis and neoangiogenesis. It has been demonstrated $[41,42]$ that knockout of TGF- $\beta$ pathway reduces Id1 expression in the gliomas that is accompanied with tumor growth assuming that Id1 is significant for tumor growth maintenance in the gliomas. The level of Id1 expression correlates with high degree of malignancy in the gliomas. This expression is not detected in the normal brain.

0ct4 is expressed in the pluripotent embryonal stem and germinal cells where it regulates self-renewal and differentiation. 0ct4 is expressed in various types of cancer, including lung cancer, where the knockout of Oct4 increases the sensitivity to chemo- and radiotherapy leading to higher apoptotic activity. Oct4 expression has been shown in the gliomas with the highest protein level in the high malignancy tumors [43]. More than $50 \%$ of the $0 \mathrm{ct} 4^{+}$cells co-express the markers of CSCs CD133 and nestin [44].

It should be noted that BTSCs essentially differ from various tumors of identical histological type and within tumor. Now the two phenotypes of BTSCs are distinguished. The first proneuronal phenotype resembles embryonal SCs and is characterized by high expression of $\mathrm{CD}_{133^{+}}$, platelet-derived growth factor receptor alpha (PDGFRa), epidermal growth factor receptor (EGFR), protein of neuronal migration doublecortin (DCX) and low CD44 expression, activation of the heat shock genes, processing of RNA and oxidative phosphorylation that indicates high proliferative and metabolic activity of these cells. BTSCs of the given phenotype differ from the fetal NSCs by more pronounced proliferative potential, reduced ability for differentiation and telomere stabilization [45]. 
Another mesenchymal transcriptional profile, analogous with the NSCs of the adults, which is localized in the subventricular zone and hippocampus, is characterized by CD133-, low PDGFRa and DCX expression and high CD44 expression. EGFR expression is noticed at given phenotype only in part of cells. The BTSCs differ from adult NSCs by reduced ability for differentiation and higher proliferative activity. In the BTSCs of this phenotype one can observe activation of the TGF- $\beta$ signaling pathway making cells a potential target for anti-TGF- $\beta$ therapy.

The phenotype of BSTCs within one tumor can differ depending on cells localization. Thus $\mathrm{CD} 133^{+}$cancer stem cells, localized in the perivascular niches, have higher expression of the Sox2, EGFR, Bmi-1 and nestin [46].

Thus, the above-given data indicate that BSTCs and NSCs have some similar properties and phenotypic markers, namely: ability for self-maintenance and sphere-formation in the serum-free medium in the presence of the growth factors rhEGF, rhFGF-b or B-27; express the markers CD133, CD44, PDGFRa, GFAP, nestin, Oct4, Sox-2, Misashi-1 and Bmi-1. The BTSCs, in contrast to NSCs, do not express early (Tuj1) and late (NeuN) neuronal markers and oligodendroglial marker Olig1. They can only express markers of the neuronal, astrocyte and oligodendroglial lines during culturing and differentiation. In addition, the NSCs, being phenotypically characterized as CD133+CD24-CD34-CD45, following their transplantation to the immune-deficient experimental animals, proliferate, migrate and differentiate according to the neuronal or glial type. However, when being transplanted to the immune-deficient experimental animals, the BTSCs form tumors histologically identical to the primary tumor from which they were obtained.

Summing up this section, we may conclude that CSCs occur in the various kinds of human tumor and have certain phenotypic resemblance by antigen markers. The malignant ectodermal tumors of the brain are the site where the NSCs can express molecular markers indicating their origin from the neural, mesenchymal and other stem cells. The CSCs markers in malignant ectodermal brain tumors can evidence about the tumor origin from neural, mesenchymal and other stem cells. At the same time no clear-cut phenotypical differences by the molecular marker between cancer and neuronal stem cells have been found so far.

\section{THERAPEUTIC APPROACHES TO THE TARGET IMPACTS ON THE CANCER STEM CELLS}

Isolation of BTSCs allows not only to study the markers of identification, antigenicity, biological properties, invasion and migration of the NSCs in the oncogenesis, but also allows examine their potential response to chemotherapy, radiotherapy and development of new therapeutic approaches to influence on this type of cells as one of the new directions with the use of BTSCs in treatment of the patients with brain tumors.

Investigations aiming to study the response of BTSCs to radiotherapy indicate that the given cells are more resistant to radiotherapy. Some mechanisms can be responsible for this phenomenon, namely: activation of DNA reparation mechanisms, reduction of DNA damage due to lesser radical production, activation of the signal pathways which are responsible for stem phenotype.

The in vitro and in vivo investigations have demonstrated that $\mathrm{CD} 133^{+}$cells versus CD133- cells have greater resistance to $\gamma$-radiation owing to early activation of cell control mechanisms. The activity of checkpoint kinases Chk1 and Chk2 in the stem cells is more increased terminating cell cycle and switching off DNA reparation mechanisms. Owing to this the NSCs avoid apoptosis and further renew proliferation resultant in tumor recurrence. In the study performed on the intracerebral xenografts in vivo Jamal M., et al. (2012) observed prevalent death of the differentiated tumor cells and increase of the glioma NSCs and glioma cancer stem cells. This was shown by an increased expression of the stem markers CD133, nestin, Notch2 and Sox2. These findings agree with the data obtained by Tamura, et al. (2010) showing 15-20- fold increase of CSCs at the recurrence of multiforme glioblastomas after irradiation of patients on the linear accelerator with summary dose of $50 \mathrm{Gr}$ and nearly $20 \mathrm{Gr}$ Gamma Knife. Such irradiation dose caused significant necrosis zones and disturbances of microvascular network in the tumor tissue. The authors noticed maintenance of CD133+ NSCs predominantly in the zone of damaged vessels that, in the authors' opinion, evidenced for the involvement of NSCs not only in the induction of tumor growth but also in the formation of vessels.

For glioma treatment the use is more frequently made of the two groups of alkyl drugs: nitrosurea derivative and temozolomide. Several investigations studying the impact of temozolomide on BCTCs have shown that treatment with this drug is accompanied with initial decrease of sphere formation of $\mathrm{CD}_{133^{+}}$cells without essential influence on cell survival. Upon treatment completion the growth of the neurospheres renewed in most cell lines along with an increase of the number of $\mathrm{CD}_{133^{+}}$cells and their tumorigenicity. Additionally it was described that $\mathrm{CD} 133^{+}$BTSCs with spheroid growth are more resistant compared with CD133- BTSCs with growth in monolayer. The mechanism mediating resistance of BTSCs to temozolomide can be the following: higher expression of MGMT and MDR1 proteins, p53 and PARP mutation. Since activation of various signaling pathways promotes maintenance of BTSCs stem phenotype, tumorigenicity suppression by temozolomide can be enhanced using inhibitors SHH, STAT3, IGF-1/ $\mathrm{PI} 3 \mathrm{~K}$ and Notch-signaling ways. The population of $\mathrm{CD} 133^{+}$cells also survives and proliferates under influence of another drug - Carmustine (BCNU). These cells have high expression of the antiapoptotic genes and genes of chemoresistance: BCRP, MDR, MRP and MGMT.

In view of the resistance of BTSCs to chemical drugs and irradiation, research has been done on various drugs which are mainly oriented at the molecular pathways of regulation of the NSCs and antibodies or antibody-based drugs to surface receptors of these cells.

The first step consists in obtaining antibodies to surface NSCs markers. An example of such approach might be influence of CD44 via specific antibody P245 to block the growth of xenotransplants of human breast cancer [48]. Besides, P245 treatment of xenotransplants of basal-cell breast cancer during tumor remission reduces the frequency of recurrence. Using the Sox6 peptide (antigen which is expressed on the surface of BTSCs), the specific T lymphocytes effectively lysed glioblastoma stem cells.

The use of anti-CD133 antibodies has brought ambiguous results. Application of monoclonal antibody against isoform AC-133-2 of CD133 molecule has had stimulating effect on tumor cells in the in vitro tests and increase of $\mathrm{CD}_{133^{+}}$cells proliferation [49]. In this connection it seems perspective to create gene-engineering constructions with monoclonal antibodies against brain tumor stem cells, capable to block proliferation of SCs.

Another direction in the strategy of treatment of oncologic patients in the context of existence CSCs is the use of constitutional expression of various factors of signal transduction, the Notch-signaling pathway in particular. Notch-signaling pathway is of crucial importance in intercellular interactions during embryonal growth, proliferation and differentiation processes. This pathway of signal transduction also plays a significant role in the processes of immune regulation and survival of neuronal stem cells and it is also involved in brain cancer formation. High correlation was observed between expression of Notch-1 and its ligands such as Delta-like- 1 and Jagged-1 and high degree of glioma malignancy $[50,51]$. The previous results showed potential regulation of CSCs Notch-signaling pathway in the medulloblastomas as well. In addition, Notch proteins are associated with CSCs, expressing marker of neuronal stem cells and nestin at gliomas. Neurosphere-formation is increased in the glioma cell lines during Notch- signaling pathway activation [52]

At present the drugs blocking Notch-signaling pathways are being developed. In particular, among various drugs undergoing preclinical trial phase the mentioning should be made of the $\gamma$-secretes 
inhibitors R04929097 (Roche) and MK0752 (Merck); and the drugs of monoclonal antibodies to various factors of Notch-signaling pathway OMP21M18, which is monoclonal antibodies against the ligand of Notch receptor DLL4. This drug is capable to produce an interfering action as a competitor with Notch receptor for the ligand.

Another important signaling cascade whose increased activity is detected in the stem cells and their malignant analogues is the Hedgehog. This signaling pathway has great importance in the regulation of embryogenesis and development of the CNS as well as in the proliferation and differentiation of the neuronal stem cells [53, 54]. Aberration of Hedgehog-pathway correlates with meduloblastoma development [55]. Besides, the Hedgehog signaling pathway is involved in CSCs self-renewal and tumorigenicity at gliomas [56]. Increase of apoptosis of the gliomas is seen during treatment by cyclonamin inhibitor or transduction of interferent RNA which inhibits proliferation and self-renewal of glioma CSCs.

It is significant that the use of Hedgehog inhibition in combination with traditional chemical drugs, temozolomide in particular, increased the death rate of BTSCs and reduced tumor cells proliferation [56]. Several investigations have demonstrated that cyclonamine treatment not only slowed tumor progression owing to its effect on the BTSCs but also increased BTSCs sensitivity to radiotherapy [57]. The abovementioned data indicate that Hedgehog-pathway is critical for BTSCs and the inhibitors targeted against it. Presently the drugs BMS83392 and XL139, which are low-molecular inhibitors of Smo receptor that is involved in the processes dependent upon Hedgehog signaling pathway, undergo clinical trials. Apart from the Smo receptor inhibitors, the trials are performed to study the drugs blocking Hedgehog effects.

The Wnt-signaling pathway regulates proliferation of stem cells and progenitor cells in the fetal ventricular zone, postnatal subventricular zone and in the hippocampus [58]. The Wnt-signaling pathway also plays an important role in the development and progressing of some medulloblastomas and gliomas. An increased expression of some components of the Wnt family was detected in the astrocytoma. The in vitro and in vivo experiments, using siRNA for Wnt2 and $\beta$-catenin, have demonstrated inhibition of cell proliferation and invasion as well as inhibition of tumor development. Furthermore, suppression of Wnt2 and $\beta$-catenin is associated with reduced PI3K/Akt expression that is associated with an interaction between $\mathrm{Wnt} / \beta$-catenin and PI3K/Akt signal cascades [59]. Expression of CD133 and Wnt-1 is elevated in the stem cells isolated from the glioblastomas. Increase of Axin, negative regulator of Wnt/ $\beta$-catenin signaling significantly inhibits glioma cells proliferation. The immune-histochemical assay showed an increase of Wnt5a in the multiforme glioblastoma in the comparison with normal tissue specimen or low degree malignancy astrocytoma. An increased Wnt5a expression was detected during proliferation in U87MG cell line. On the contrary, Wnt5a suppression due to RNA interference reduces proliferation of this culture and decreases tumorigenicity in vivo [60].

\section{CONCLUSION}

Thus currently presented literature data describe the presence of intracerebral cancer cells in tissues, namely: the brain tumor stem cells capable for self-renewal and self-maintenance and morphologically identical to the neurospheres, which are formed by normal neural stem cells and capable upon their injection to immune-deficient mice induce formation of tumors histologically identical to the primary tumor of their origin. These cells express many antigens, including CD133, CD105, CD90, CD15, Nanog, Oct3/4, CXCR4 (CD184), NF and GAPDH as well as antigens to cancer stem cells.

These cells are characterized by increased invasion, radio- and chemoresistance. Their further investigation will allow disclose their involvement in the formation and recurrence of the brain malignant tumors and to develop methods of purpose-designed therapy aiming at increase of effectiveness of standard therapy for patients with brain tumors.

Interest to study brain tumor stem cells as well as cancer stem cells of other organs and tissues is not becoming lesser with years. In current research of the CSCS we can outline several research directions, namely: (a) assessment of CSCs level in tumors of various origin; (b) prognostication of the process of malignization based on brain tumor SCs; (c) origin and biological activity of the CSCs; (d) assessment of transformation and change of the intracellular signaling pathways providing proliferation and apoptosis of these cells; (e) search for new means to block and activate CSCs, increase their sensitivity to radiotherapy, etc. This list can be continued but unfortunately the data obtained of today cannot be clinically used.

At the present stage of investigation of the CSCs and specifically brain tumor stem cells such as glioblastomas and meduloblastomas, it is essential to clarify whether:

1. The brain tumor stem cells are modified normal neural stem cells or whether they are a reverse mutational differentiation of the mature glial cells under influence of cancerogenes and viruses to the level of stem cell precursors, as has been proposed in the hypothesis of Junier M. and Sharif A. or probably these mechanisms run simultaneously.

2. The availability of a considerable number of various phenotypical membrane and intracellular markers on both BTSCs and NSCs determines a need to single out the specific markers characteristic only of the CSCs and only of the NSCs, allowing further not only discern them but also develop, on the basis of monoclonal antibodies, the specific drugs against these cells.

3. It is necessary to identify one or two main intracellular signaling pathways in the CSCs responsible for proliferation and radioresistance, which will become an object of deeper investigation.

Current and future research should offer new opportunities not only for extending our theoretical knowledge about the oncogenesis and malignancy development but also for anticancer drug discovery including brain tumor-targeted drugs.

\section{REFERENCES}

1. Bonnet D, Dick JF. Human acute myeloid leukemia is organized as a hierarchy that originates from a primitive hematopoietic cell. Nat Med. 1997; 3:730-737.

2. Reya T, Morrison SJ, Clarke IL. Stem cells, cancer, and cancer stem cells. Nature. 2001; 414:105-111.

3. Clevers $H$. The cancer stem ceil: Premises, promises and challenges. Nat. Med. 2011; 17:313-319.

4. Suetsugu A, Nagaki M, Aokil H. Characterization of CD133+ hepatocellular carcinoma cells as cancer stem/progenitor cells. Biochem Biophys Res Commun. 2006; 29(4):820-824. 
5. Yuan X, Curtin J, Xiong Y, et al. Isolation of cancer stem cells from adult glioblastoma multiforme. Oncogene. 2004; 16(23):9392-9400.

6. Vescovi AL, Gall R, Reynolds BA. Brain tumour stem cells. Nat. Rev.Canser. 2006; 6:425-436.

7. Dalerba P, Cho RW, Clarke MF. Cancer stem cells: Models and concepts. Ann. Rev. Med. 2007; 58:267-284.

8. Wicha MS. Cancer stem cells and metastasis: Lethal seeds. Clin. Cancer. Res. 2006; 12:5606-5607.

9. Shiras $A$, Chettiar ST, Shepal V. Spontaneous transformation of human adult nontumorogenic stem sells to cancer stem cells is driven by gemonic instability in a human model of glioblastoma. Stem Cells. 2007; 25(6):1478-1489.

10. Masui K, Suzuki SO, Torisu R. Glial progenitors in the brainstem give rise to malignant gliomas by platelet-derived growth factor stimulation. Glia. 2010; $\mathbf{5 8 ( 9 )}$ 1050-1065.

11. Junier MP, Sharif A. Instability of cell phenotype and tumor initiating cells in gliomas. Biol. Aujordhui (French). 2011; 205(1):63-74.

12. Galli R, Binda E, Orfanelli U. Isolation and characterization of tumorigenic, stem-like neural precursors from human glioblastoma. Cancer Res. 2004; 1(19):7011-7021.

13. Singh SK, Clarke ID, Terasaki M, et al. Identification of a cancer stem cell in human brain tumors. Cancer Res. 2003; 63:5821-5828.

14. Zhang M, Song T, Yang L, et al. Nestin and CD-133: valuable stem cell-specific markers for determing clinical outcome of glioma patients. J. Exp Clin Cancer Res. 2008; 24(27):85-92.

15. Pallini RL, Ricci-Vitiani L, Montano N. Expression of the stem cell marker CD133 in recurrent glioblastoma and its value for prognosis. Cancer. 2011; 117(1):162-174.

16. Zeppernick F, Ahmadi R, Campos B, et al. Stem cell marker CD133 affects clinical outcome in glioma patients. Clin Cancer Res. 2008; 14:123-129.

17. Ma YH, Mentlein R, Knerlich F, et al. Expression of stem cell markers in human astrocytomas of different WHO grades. J. Neurooncol. 2008; 86:31-45.

18. Kim KJ, Lee KH, Kim HS, et al. The presence of stem cell marker-expressing cells is not prognostically significant in glioblastomas. Neuropathology. 2011; 31: 494-502.

19. Lee J, Kotliarova $S$, Kotliarov $Y$, et al. Tumor stem cells derived from glioblastomas cultured in bFGF and EGF more closely mirror the phenotype and genotype of primary tumors than do serum-cultured cell lines. Cancer Cell. 2006; 9:391-403.

20. Bo Qio, Zhang D, Tao J. A simplified and modified procedure to culture brain glioma stem cells from clinical specimens. Oncol Lett. 2012; 1:50-54.

21. Beier D, Hau P, Proescholdt M. CD133+ and CD133- glioblastoma-derived cancer stem cells show differential growth characteristics and molecular profiles. Cancer Res. 2007; 17(9):4010-4015.

22. Choung YK, Toh TB, Zaiden N. Cryopreservation of neurosphtres derived from human glioblastoma multiforme. Stem Cells. 2009; 27(1):29-39.

23. Read TA, Fogarty MP, Markant SL, et al. Identification of CD15 as a marker for tumor-propagating cells in a mouse model of medulloblastoma. Cancer Cell. 2009; 15:135-147.

24. Son MJ, Woolard K, Nam DH, et al. SSEA-1 is an enrichment marker for tumorinitiating cells in human glioblastoma. Cell Stem Cell. 2009; 4:440-452.

25. He J, Liu Y, Zhuet T, et al. CD90 is identified as a marker for cancer stem cells in primary high-grade gliomas using tissue microarrays. Mol. Cell Proteomics. 2010; 11(6):M111.010744.

26. Tchoghandjian A, Baeza N, Colin C, et al. A2B5 cells from human glioblastoma have cancer stem cell properties. Brain Pathol. 2010; 20:211-221.

27. Piepmeier JM, Fried I, Makuch R. Low-grade astrocytomas may arise from different astrocyte lineages. Neurosurgery. 1993; 33:627-632.

28. Chinnaiyan $P$, Wang $M$, Rojiani $A M$, et al. The prognostic value of nestin expression in newly diagnosed glioblastoma: report from the Radiation Therapy Oncology Group. Radiat Oncol. 2008; 3:32.

29. Wan F, Herold-Mende C, Campos B, et al. Association of stem cell-related markers and survival in astrocytic gliomas. Biomarker. 2011; 16:136-143.

30. Arai $\mathrm{H}$, Ikota $\mathrm{H}$, Sugawara $K$, et al. Nestin expression in brain tumors: its utility for pathological diagnosis and correlation with the prognosis of high-grade gliomas. Brain Tumor Pathol. 2012; 29:160-167.

31. Kim KJ, Lee KH, Kim HS, et al. The presence of stem cell marker-expressing cells is not prognostically significant in glioblastomas. Neuropathology. 2011; 31: 494-502.

32. Gangemi RM, Griffero F, Marubbi D, et al. SOX2 silencing in glioblastoma tumor-initiating cells causes stop of proliferation and loss of tumorigenicity. Stem Cells. 2009; 27:40-48.

33. Phi JH, Park SH, Kim SK, et al. Sox2 expression in brain tumors: a reflection of the neuroglial differentiation pathway. Am J Surg Pathol. 2008; 32:103-112.

34. Wan F, Herold-Mende C, Campos B, et al. Association of stem cell-related markers and survival in astrocytic gliomas. Biomarkers. 2011; 16:136-143.

35. Mishima $K$, Kato $Y$, Kaneko $M K$, et al. Increased expression of podoplanin in malignant astrocytic tumors as a novel molecular marker of malignant progression. Acta Neuropathol. 2006; 111:483-488.

36. Venugopal C, Li N, Wang X, et al. Bmi1 marks intermediate precursors during differentiation of human brain tumor initiating cells. Stem Cell Res. 2012; 8:141-153.

37. Hayry V, Tynninen O, Haapasalo HK, et al. Stem cell protein BMI-1 is an independent marker for poor prognosis in oligodendroglial tumour. J. Neuropathol Appl Neurobiol. 2008; 34: 555-563.

38. Hemmati HD, Nakano I, Lazareff JA, et al. Cancerous stem cells can arise from pediatric brain tumors. Proc Natl Acad Sci U S A. 2003; 100:15178-15183.

39. Ma YH, Mentlein R, Knerlich F, et al. Expression of stem cell markers in human astrocytomas of different WHO grades. J Neurooncol. 2008; 86:31-45.

40. Kanemura Y, Mori K, Sakakibara S, Fujikawa $\mathrm{H}$, et al. Musashi1, an evolutionarily conserved neural RNA-binding protein, is a versatile marker of human glioma cells in determining their cellular origin, malignancy, and proliferative activity. Differentiation. 2001; 68:141-152.

41. lavarone A, Lasorella A. ID proteins as targets in cancer and tools in neurobiology. Trends Mol Med. 2006; 12:88-594.

42. Phi JH, Kim JH, Eun KM, et al. Upregulation of SOX2, NOTCH1, and ID1 in supratentorial primitive neuroectodermal tumors: a distinct differentiation pattern from that of medulloblastomas. J Neurosurg Pediatr. 2010; 5:608-614.

43. Du Z, Jia D, Liu S, et al. Oct4 is expressed in human gliomas and promotes colony formation in glioma cells. Glia. 2009; 57:724-733.

44. Guo YL, Liu S, Wang P, et al. Expression profile of embryonic stem cell-associated genes Oct4, Sox2 and Nanog in human gliomas. Histopathology. 2011; 59(4):763775 .

45. Lottaz C, Beier D, Mayer K, et al. Transcriptional profiles of CD133+ and CD133- glioblastoma - derived cancer stem cell lines suggest different ctll of origin. Canser stem cell lines suggest different cell of origin Cancer Res. 2010; 70(5):2030-2040.

46. Christen K, Shirder HD, Kristensen BW, et al. CD133+ niches and signle in glioblastoma have different phenotypes . J.Neurooncol. 2011; 104(1):129-143.

47. Jamal $M$, Rath $B N$, Tsang $P$, et al. The brain microenvironment preferentially enhances the radioresistance of CD 133(+) glioblastoma stem-like. Neoplasia. 2012; 14:150-158.

48. He J, Liu Y, Lubman D. Targeting glioblastoma stem cells: cell surface markers. Curr Med Chem. 2012; 19(35):6050-6055. 
49. Lisyany NI. Immunologija i immunoterapija zlokachestvennyh gliom golovnogo mozga. [Immunology and Immunotherapy of malignant gliomas of the brain]. Serija «Nejroimmunologija». Kiev: Interservis - Series «Neuroimmunology». Kiev: Interservice. 2011; 5: 240 p.

50. Purow MW, Haque BW, Noel RM, et al. Expression of Notch-1 and its ligands, Delta-like-1 and Jagged-1, is critical for glioma cell survival and proliferation. Cancer Res. 2005; 65(6):2353-2563.

51. Fan X, Mikolaenko I, Elhassan I, et al. Notch1 and notch2 have opposite effects on embryonal brain tumor growth. Cancer Res. 2004; 64(21):7787-7793.

52. Zhang XP, Zheng G, Zou L, et al. Notch activation promotes cell proliferation and the formation of neural stem cell-like colonies in human glioma cells. Mol Cell Biochem. 2008; 307:101-108.

53. Park Y, Rangel C, Reynolds MM, et al. Drosophila perlecan modulates FGF and hedgehog signals to activate neural stem cell division. Dev Bio. 2003; 253(2):247-257.

54. Becher OJ, Hambardzumyan D, Fomchenko El, et al. Gli activity correlates with tumor grade in platelet-derived growth factor-induced gliomas. Cancer Res. 2008; 68(7):2241-2249.

55. Dahmane N, Sanchez P, Gitton Y, et al. The Sonic Hedgehog-Gli pathway regulates dorsal brain growth and tumorigenesis. Development . $2001 ;$ 128:5201-5212.

56. Clement $V$, Sanchez $P$, Tribolet N, et al. HEDGEHOG-GLI1 signaling regulates human glioma growth, cancer stem cell self-renewal, and tumorigenicity. Curr Biol: CB. 2007; 17:165-172.

57. Bar EE, Chaudhry A, Lin A, et al. Cyclopamine-mediated hedgehog pathway inhibition depletes stem-like cancer cells in glioblastoma. Stem Cells. 2007; 25: 2524-2533.

58. Lie DC, Colamarino SA, Song HJ, et al. Wnt signalling regulates adult hippocampal neurogenesis. Nature. 2005; 437(7063):1370-1375.

59. Pu P, Zhang Z, Kang C, et al. Downregulation of Wnt2 and betacatenin by siRNA suppresses malignant glioma cell growth. Cancer Gene Ther. $2009 ; 16(4): 351-361$.

60. Yu JM, Jun ES, Jung JS, et al. Role of Wnt5a in the proliferation of human glioblastoma cells. Cancer Lett. 2007; 257:172-181.

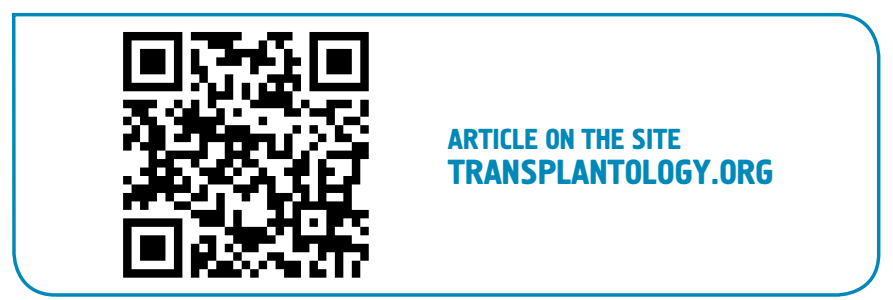

The authors indicate no potential conflicts of interest. 